\title{
CODIMENSION TWO SUBMANIFOLDS OF POSITIVE CURVATURE
}

\author{
JOHN DOUGLAS MOORE
}

\begin{abstract}
In this note it is proven that a compact connected $n$-dimensional Riemannian manifold of positive curvature, isometrically immersed in $(n+$ 2)-dimensional Euclidean space, is a homotopy sphere if $n>3$; hence it is homeomorphic to a sphere if $n>5$.
\end{abstract}

1. Introduction. Among the most beautiful applications of Morse theory are those which relate topology to curvature of Riemannian manifolds. We are interested in applying Morse theory to a slightly different problem: that of determining relationships between topology and curvature of submanifolds of low codimension in Euclidean space.

Let us recall some known facts. According to Alan Weinstein [8], an $n$-dimensional Riemannian manifold $M^{n}$ with positive sectional curvatures, which is isometrically immersed in $(n+2)$-dimensional Euclidean space $E^{n+2}$, must have positive curvature operators. This implies, according to a theorem of Daniel Meyer [3], that if in addition, $M^{n}$ is compact and connected, its universal cover must be a rational homology sphere. In this note, we will use an inequality of Chang-shing Chen to show that in fact $M^{n}$ must be a homotopy sphere:

THEOREM. Let $M^{n}$ be a compact connected n-dimensional Riemannian manifold with positive sectional curvatures, which is isometrically immersed in $E^{n+2}$. Then if $n \geqslant 3, M^{n}$ is a homotopy sphere. Hence if $n \geqslant 5, M^{n}$ is homeomorphic to a sphere.

The last sentence follows from the rest of the theorem by means of the $h$-cobordism theorem $[5, \S 9]$.

For example, our theorem implies that if $M^{n}$ is a spherical space form which admits an isometric immersion in $E^{n+2}$, then $M^{n}$ must be simply connected. (Compare [6, Propositions 4 and 5].)

2. The integrated Morse inequalities. We consider an immersion $f$ from a compact $n$-dimensional manifold $\boldsymbol{M}^{n}$ into $N$-dimensional Euclidean space $E^{N}$. Let $S^{N-1}$ denote the unit sphere at the origin of $E^{N}$. For each $u \in S^{N-1}$,

Received by the editors October 2, 1977.

AMS (MOS) subject classifications (1970). Primary 53C40, Secondary 58E99.

Key words and phrases. Submanifolds of positive curvature, Morse theory, isometric immersions.

'Partially supported by NSF grant MCS 77-01843. 
we have a height function

$$
h_{u}: M^{n} \rightarrow \mathbf{R} \text { defined by } h_{u}(p)=f(u) \cdot p,
$$

where the dot denotes the usual Euclidean dot product. For almost all $u, h_{u}$ is a Morse function. For each $u \in S^{N-1}$, let $\mu_{k}(u)$ denote the number of nondegenerate critical points of index $k$ of the height function $h_{u}$. Following Kuiper [2], we consider the total curvature of index $k$,

$$
\tau_{k}=\frac{1}{\operatorname{vol}\left(S^{N-1}\right)} \int_{S^{N-1}} \mu_{k}(u)
$$

We can regard $\tau_{k}$ as the "average number of critical points of index $k$." Since replacing $u$ by $-u$ changes critical points of index $k$ into critical points of index $n-k$, we see that $\tau_{n-k}=\tau_{k}$.

We will apply the Morse inequalities $[4, \S 5]$ to the height functions $h_{u}$. Choose a field $F$ and set $\beta_{k}=\operatorname{dim} H_{k}\left(M^{n} ; F\right)$. If $u$ is an element of $S^{N-1}$ such that $h_{u}$ is a Morse function, then

$$
\beta_{k} \leqslant \mu_{k}(u), \quad \beta_{1}-\beta_{0} \leqslant \mu_{1}(u)-\mu_{0}(u), \ldots
$$

We average over $S^{N-1}$ to obtain "Morse inequalities" which relate Betti numbers to total curvatures:

$$
\beta_{k} \leqslant \tau_{k}, \quad \beta_{1}-\beta_{0} \leqslant \tau_{1}-\tau_{0}, \ldots
$$

In particular, if $M^{n}$ is connected, so that $\beta_{0}=1$,

$$
\beta_{1} \leqslant \tau_{1}-\tau_{0}+1 \text {. }
$$

If $M^{n}$ is orientable over $F$, then by Poincaré duality,

$$
\beta_{n-1} \leqslant \tau_{n-1}-\tau_{n}+1 \text {. }
$$

(1) and (2), together with $\beta_{k} \leqslant \tau_{k}$, yield

$$
\beta_{1}+\cdots+\beta_{n-1} \leqslant\left(\tau_{1}+\cdots+\tau_{n-1}\right)-\left(\tau_{0}+\tau_{n}\right)+2 .
$$

3. Proof of the theorem. We will make use of an inequality of Chen, which holds when $M^{n}$ has positive curvature and $N=n+2$ :

$$
\tau_{1}+\cdots+\tau_{n-1}<\tau_{0}+\tau_{n} .
$$

This inequality follows from Proposition 2.5 of [1]. (Note that due to a typographical error, the inequality as stated in [1] is reversed.) It follows immediately from (3) and (4) that

$$
\beta_{1}+\cdots+\beta_{n-1}<2,
$$

so long as $M^{n}$ is orientable over the field $F$.

We now show that under the hypotheses of our theorem, $M^{n}$ must be simply connected. If not, $\pi_{1}\left(M^{n}\right)$ contains a subgroup isomorphic to $\mathbf{Z}_{p}$ for some prime number $p$. Let $\tilde{M}^{n}$ be the Riemannian covering of $M^{n}$ corresponding to this subgroup. It is easy to see that $\tilde{M}^{n}$ is compact and orientable over $\mathbf{Z}_{p}$. Moreover, the composition of the covering projection with the isometric immersion $M^{n} \rightarrow E^{n+2}$ yields an isometric immersion $\tilde{M}^{n} \rightarrow E^{n+2}$. 
If we set $\beta_{k}=\operatorname{dim} H_{k}\left(\tilde{M}^{n} ; \mathbf{Z}_{p}\right)$, we find that $\beta_{1}=\beta_{n-1}=1$, contradicting (5). Thus $M^{n}$ must be simply connected.

Next we show that $M^{n}$ has the same homology groups over the integers as a sphere. Since the work of Weinstein and Meyer shows that $M^{n}$ is a rational homology sphere, we need only show that $M^{n}$ has no torsion. But if $H_{k}\left(M^{n}\right.$; Z) contains torsion for some $k, 0<k<n$, then $H_{k}\left(M^{n} ; \mathbf{Z}_{p}\right) \neq 0$ for some prime $p$, and by Poincaré duality $H_{n-k}\left(M^{n} ; \mathbf{Z}_{p}\right) \neq 0$. This contradicts (5) unless $k=n / 2$. Thus if $M^{n}$ is not a homology sphere over the integers, it must be even-dimensional, say $n=2 m$, and all its torsion must lie in $H_{m}\left(M^{n}\right.$; Z). But again by Poincaré duality (torsion $H_{m}\left(M^{n} ; Z\right) \cong$ torsion $H_{m-1}\left(M^{n}\right.$; Z)), this is impossible.

Thus $M^{n}$ is simply connected and has the homology of a sphere, so by the Hurewicz isomorphism theorem, $\pi_{i}\left(M^{n}\right)=0$ for $0<i<n$ and $\pi_{n}\left(M^{n}\right) \cong \mathbf{Z}$. Let $g: S^{n} \rightarrow M^{n}$ be a representative for a generator of $\pi_{n}\left(M^{n}\right) . g$ is a mapping between simply connected $\mathrm{CW}$ complexes which induces isomorphisms of homology groups. Hence by [7, p. 399, Theorem 9 and p. 405, Corollary 24], $g$ is a homotopy equivalence and $M^{n}$ is indeed a homotopy sphere. This finishes the proof of the theorem.

\section{REFERENCES}

1. C. S. Chen, On tight isometric immersions of codimension two, Amer. J. Math. 94 (1972), 974-990.

2. N. H. Kuiper, Minimal total absolute curvature for immersions, Invent. Math. 10 (1970), 209-238.

3. D. Meyer, Sur les variétés riemanniennes à opérateur de courbure positif, C. R. Acad. Sci. Paris 272 (1971), 482-485.

4. J. Milnor, Morse theory, Ann. of Math. Studies, no. 51, Princeton Univ. Press, Princeton, N. J., 1963.

5. , Lectures on the h-cobordism theorem, Math. Notes, Princeton Univ. Press, Princeton, N. J., 1965.

6. J. D. Moore, Submanifolds of constant positive curvature. I, Duke Math. J. 44 (1977), 449-484.

7. E. H. Spanier, Algebraic topology, McGraw-Hill, New York, 1966.

8. A. Weinstein, Positively curved n-manifolds in $\mathbf{R}^{n+2}$, J. Differential Geometry 4 (1970), 1-4.

Department of Mathematics, University of California, Santa Barbara, California 93106 